\section{Prevalência de sedentarismo e fatores associados em adolescentes de 10-12 anos de idade}

\author{
Prevalence of sedentary lifestyle and associated \\ factors in adolescents 10 to 12 years of age
}

Pedro Curi Hallal 1

Andréa Dâmaso Bertoldi 1

Helen Gonçalves 1

Cesar Gomes Victora ${ }^{1}$

\footnotetext{
1 Programa de Pós-graduação em Epidemiologia

Universidade Federal de Pelotas, Pelotas, Brasil.

Correspondência P. C. Hallal

Programa de Pós-graduação em Epidemiologia, Universidade Federal de Pelotas.

Av. Duque de Caxias 250,

3o andar, Pelotas, $R S$ 96030-002, Brasil. prchallal@terra.com.br
}

\section{Abstract}

Physical activity in adolescence is associated with several health benefits, including a direct influence on adolescent morbidity and an indirect effect on adult health mediated by physical activity levels in adulthood. This study assessed the prevalence of sedentary lifestyle and associated variables in 4,452 adolescents aged 10-12 years, belonging to the 1993 Pelotas Birth Cohort Study, representing $87.5 \%$ of the original cohort. Sedentary lifestyle, defined as $<300 \mathrm{~min}$ utes per week of physical activity, was reported by 58.2\% (95\%CI: 56.7-59.7) of the cohort. In the multivariate analysis, sedentary lifestyle was positively associated with female gender, socioeconomic status, maternal physical inactivity, and television viewing, but inversely correlated with time spent playing videogames. Adolescents with low socioeconomic status were more likely to walk or bicycle to and from school. Effective strategies against sedentary lifestyle in adolescence are needed because of its high prevalence and association with physical inactivity in adulthood.

Motor Activity; Physical Fitness; Adolescent; Exercise

\section{Introdução}

A prática adequada de atividade física na adolescência traz vários benefícios para a saúde física e mental, seja por meio de uma influência direta sobre a morbidade na própria adolescência ou por uma influência mediada pelo nível de atividade física na idade adulta $1,2,3,4$. Embora a maioria das doenças associadas ao sedentarismo somente se manifeste na vida adulta, é cada vez mais evidente que seu desenvolvimento se inicia na infância e adolescência 5 . Sendo assim, o estímulo à prática de atividade física desde a juventude deve ser uma prioridade em saúde pública.

Apesar dessas evidências, a prevalência de sedentarismo ainda é muito alta, tanto em países ricos 6,7 quanto naqueles de renda média ou baixa 8,9 . Ainda mais preocupantes, são as evidências de que a aptidão física (medida pela resistência cardiorrespiratória) de adolescentes vem apresentando tendências de declínio em algumas populações 10 .

No Brasil, há poucos estudos de base populacional ou escolar sobre atividade física em adolescentes. Em um estudo transversal realizado na Cidade de Niterói com adolescentes de 14-15 anos, $85 \%$ dos meninos e $94 \%$ das meninas foram classificados como sedentários - escore abaixo de três em uma escala que variava de zero a cinco pontos; a escala englobava atividades esportivas realizadas no tempo de la- 
zer 11. Em adolescentes de 15 a 19 anos residentes na zona urbana da Cidade de Pelotas, a prevalência de sedentarismo - menos de vinte minutos de atividade física pelo menos três vezes por semana - foi de $22 \%$ nos meninos e $55 \%$ nas meninas 12 . Outros estudos nacionais entre adolescentes também relatam prevalências muito variadas 13,14 . É provável que a utilização de instrumentos e definições de sedentarismo bastante distintas 1 explique pelo menos parte dessas diferenças, sendo a padronização de instrumentos e pontos de corte essenciais para a comparabilidade de resultados.

O objetivo deste estudo foi descrever os níveis de atividade física em diversos domínios (deslocamento, escola, lazer) e determinar a prevalência de sedentarismo e fatores associados entre adolescentes de 10-12 anos de idade, participantes do Estudo de Coorte de Nascimentos de 1993 em Pelotas, Rio Grande do Sul.

\section{Materiais e métodos}

No ano de 1993, os partos hospitalares (mais de 99\% de todos os partos) ocorridos na Cidade de Pelotas foram monitorados 15 . Crianças nascidas com vida cujas famílias residiam na zona urbana da Cidade de Pelotas ( $\mathrm{N}=5.249$ ) foram incluídas no estudo. Amostras dessas crianças foram visitadas em diferentes idades. Em 2004 iniciou-se um novo acompanhamento buscando entrevistar todos os jovens pertencentes ao estudo de coorte. O trabalho de campo foi iniciado em julho de 2004 e encerrado em março de 2005.

Para auxiliar na localização dos adolescentes, diversas estratégias de busca foram utilizadas. As duas principais incluíram um censo em todas as escolas do município, e um censo domiciliar cobrindo os cerca de 100 mil domicílios da cidade. Com essas estratégias, cerca de 4.700 participantes da coorte foram identificados. Aqueles que não foram localizados dessas formas foram buscados nos endereços obtidos nas visitas anteriores, registros hospitalares, instituições de cuidado de menores, entre outros. O Sistema de Informações de Mortalidade (SIM) também foi revisado em busca de óbitos.

O instrumento para coleta de dados incluiu entrevista com a mãe e com o adolescente. O questionário das mães incluiu perguntas sobre condição sócio-econômica da família (Critério de Classificação Econômica Brasil - dividido em cinco níveis, de A a E, em ordem decrescente de nível sócio-econômico 16), tipo de moradia (casa ou apartamento) e prática de atividades físicas no lazer nos sete dias anteriores à entrevista Foram classificadas como inativas as mães que não praticaram atividade física no lazer na última semana. As mães e adolescentes foram pesados e medidos com balanças portáteis (SECA, Birmingham, Inglaterra; precisão de $100 \mathrm{~g}$ ) e antropômetros de alumínio (precisão de 1mm). O Índice de Massa Corporal (IMC) materno foi calculado e dividido em três categorias (normal: < 25kg/m²; sobrepeso: $25-29,9 \mathrm{~kg} / \mathrm{m}^{2}$; obesidade: $\geq 30 \mathrm{~kg} / \mathrm{m}^{2}$ ). A idade materna no momento do parto foi incluída nas análises.

O questionário dos adolescentes incluiu diversas informações sobre atividade física: o número de aulas semanais de educação física na escola; o modo de deslocamento para a escola e o tempo gasto; o tempo total despendido em atividade física no lazer, incluindo atividades com instrutor dentro e fora da escola (escolinhas, equipes esportivas, dança, ginástica) ou sem instrutor; e o percentual de adolescentes que participam em cada tipo de atividade física no lazer. Sedentarismo foi definido como menos de trezentos minutos por semana de atividade física no deslocamento ou no lazer conforme recomendação atual para adolescentes 17. Aulas de educação física não foram incluídas uma vez que a intensidade destas atividades é usualmente muito baixa 18 , tanto nas escolas públicas quanto nas particulares.

Os questionários utilizados neste estudo estão disponíveis na página eletrônica do Centro de Pesquisas Epidemiológicas da Universidade Federal de Pelotas (http://www.epidemio-ufpel.org.br/_projetos_de_pesquisas/resultado.php?id_resultado=3).

Os adolescentes informaram o nome da escola que freqüentavam (sendo estas classificadas posteriormente em municipais, estaduais ou privadas), assim como o tempo diário médio despendido assistindo à televisão, jogando vídeo game ou utilizando o computador. O IMC e o tempo diário médio assistindo à televisão foram analisados sob a forma de tercis. O tempo de uso de vídeo-game e computador também foi classificado em três grupos: não usuários; menos de uma hora por dia; uma hora ou mais por dia.

Com 4.451 adolescentes entrevistados, foi possível estimar a prevalência de sedentarismo com uma margem de erro de 1,5 ponto percentual e nível de confiança de 95\%. Para estudo dos fatores associados ao sedentarismo, a amostra permitiu detectar como significativas razões de prevalência de 1,10 ou maiores para exposições afetando de $5 \%$ a $50 \%$ da população estudada, com $80 \%$ de poder e $95 \%$ de nível de confiança.

As entrevistadoras foram treinadas durante quarenta horas, além de terem as mensurações 
de peso e altura padronizadas; os erros técnicos das medidas foram avaliados, estando dentro dos limites do National Center for Health Statistics dos Estados Unidos 19. As sessões de padronização foram repetidas a cada dois meses durante o trabalho de campo. Um questionário resumido foi repetido para $10 \%$ dos entrevistados como controle de qualidade. Dois estudos piloto foram realizados, sendo o primeiro utilizado principalmente para teste de compreensão das perguntas e o segundo para avaliação das entrevistadoras em situação prática.

Os dados foram duplamente digitados, com checagem automática de consistência e amplitude. A análise descritiva incluiu cálculos de proporções e intervalos de confiança de $95 \%$ (IC95\%). Na análise bruta, a prevalência de cada desfecho foi calculada para as categorias das variáveis independentes. A significância foi avaliada pelo teste do qui-quadrado (heterogeneidade ou tendência linear). Na análise multivariável, utilizou-se regressão de Poisson 20, com ordem de entrada das variáveis conforme modelo hierárquico de causalidade, determinado a priori 21 . O modelo utilizado incluiu no primeiro nível as variáveis: sexo, nível sócioeconômico, tipo de moradia e tipo de escola. No segundo nível, foram utilizados o IMC e o nível de atividade física no lazer da mãe. No terceiro, estavam as demais variáveis referentes ao adolescente. O nível de significância usado foi de $5 \%$ e todos os testes foram bicaudais.

O projeto do estudo foi aprovado pelo Comitê de Ética em Pesquisa da Faculdade de Medicina da Universidade Federal de Pelotas. As mães ou responsáveis assinaram um termo de consentimento concordando com a participação dos jovens no estudo.

\section{Resultados}

Entre os 5.249 participantes da coorte, 141 foram detectados no SIM. Dentre os 5.108 restantes, 4.451 foram entrevistados, os quais, se somados aos óbitos, representam $87,5 \%$ da coorte original. A Tabela 1 apresenta o percentual de localização conforme variáveis demográficas e sócio-econômicas. Não houve diferenças significativas no percentual de localização conforme sexo do adolescente ou idade materna, mas houve maior percentual de perdas nas famílias de nível sócio-econômico mais alto.

Apenas $0,7 \%$ dos meninos e $0,6 \%$ das meninas não freqüentavam a escola. Entre os 4.413 $(99,2 \%)$ adolescentes que freqüentavam escola, $72,8 \%$ utilizam regularmente um modo de transporte ativo (caminhada ou bicicleta) para
Tabela 1

Número de crianças na coorte original e percentual localizado aos 10-12 anos conforme variáveis demográficas e sócio-econômicas.

\begin{tabular}{|c|c|c|}
\hline Variável & $\begin{array}{c}\text { Coorte original } \\
\text { (N) }\end{array}$ & $\begin{array}{l}\text { Percentual localizado } \\
10-12 \text { anos }^{\star}\end{array}$ \\
\hline Sexo & & $p=0,18^{\star \star}$ \\
\hline Masculino & 2.580 & 86,9 \\
\hline Feminino & 2.667 & 88,1 \\
\hline Renda familiar (salários mínimos) & & $p<0,001^{* *}$ \\
\hline$<1,1$ & 967 & 88,3 \\
\hline $1,1-3,0$ & 2.260 & 88,7 \\
\hline $3,1-6,0$ & 1.204 & 88,9 \\
\hline $6,1-10,0$ & 433 & 79,9 \\
\hline$>10,0$ & 385 & 82,6 \\
\hline Idade materna em 1993 (anos) & & $p=0,19 * \star$ \\
\hline$<20$ & 915 & 87,7 \\
\hline $20-34$ & 3.956 & 87,1 \\
\hline$\geq 35$ & 577 & 89,8 \\
\hline $\begin{array}{l}\text { Escolaridade materna } \\
\text { em } 1993 \text { (anos) }\end{array}$ & & $p<0,001^{\star *}$ \\
\hline $0-4$ & 1.472 & 88,1 \\
\hline $5-8$ & 2.424 & 89,9 \\
\hline$\geq 9$ & 1.350 & 82,5 \\
\hline Total & 5.249 & 87,5 \\
\hline
\end{tabular}

* Inclui 141 óbitos conhecidos:

** Teste do qui-quadrado.

comparecer até a mesma. Entre esses, o tempo médio despendido com deslocamento foi de 22,3 minutos (DP 14,8), com um valor máximo de duzentos minutos diários no deslocamento de ida e volta. Entre os meninos, 12,3\% relataram o uso de carro ou moto no deslocamento para a escola, $12,1 \%$ ônibus, $67,2 \%$ caminhada e $8,4 \%$ bicicleta. Já entre as meninas, os respectivos percentuais foram $12,1 \%, 13,1 \%, 70 \%$ e $4,6 \%$.

A Figura 1 mostra os modos de transporte para a escola conforme nível sócio-econômico. O deslocamento a pé associou-se negativamente com o nível sócio-econômico, sendo o percentual de caminhada entre os adolescentes do nível E seis vezes maior do que o observado no nível A. A utilização de carro ou moto mostrou tendência contrária, sendo que os adolescentes do nível A utilizaram carro ou moto como modo de transporte 63 vezes mais do que os do nível E. A utilização de ônibus mostrou uma associação em forma de "U" invertido com o nível sócio-econômico. O uso de bicicleta como modo de transporte para a escola associouse negativamente com o nível sócio-econômi- 
Figura 1

Modos de deslocamento para a escola conforme nível sócio-econômico.

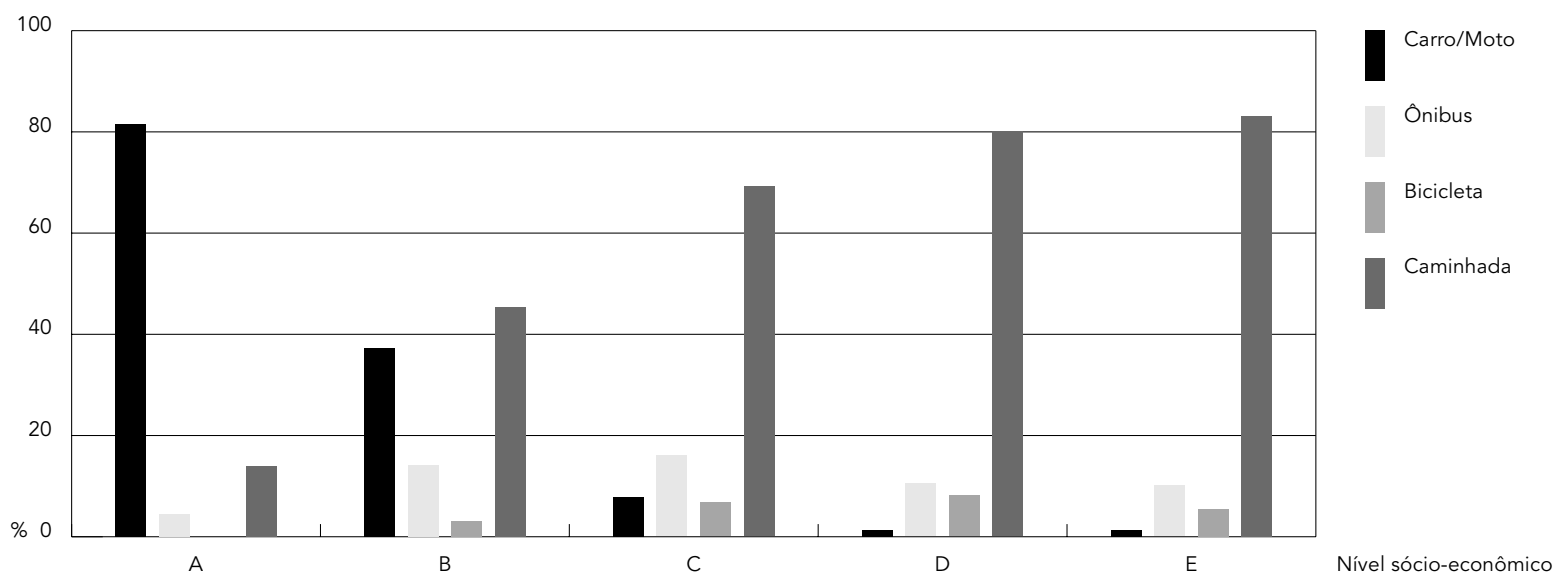

co, mas houve um discreto desvio da tendência no nível E.

Para cada modalidade de atividade física praticada no lazer nos sete dias anteriores à entrevista, os adolescentes informaram o número de vezes que realizaram aquela atividade e sua duração. A Figura 2 mostra as atividades praticadas pelos meninos e pelas meninas. Entre os meninos, as atividades mais praticadas (em ordem) foram futebol, voleibol e caçador (ou jogo de pega-pega com bola), enquanto que entre as meninas foram voleibol, caçador (ou jogo de pega-pega com bola) e futebol. As diferenças no percentual de participação em cada atividade conforme o sexo foram avaliadas, sendo que as seguintes atividades foram praticadas com freqüência significativamente maior entre os meninos do que entre as meninas: futebol, jogo de taco, futsal, lutas, atletismo, basquete e natação. A prática de ginástica, dança, voleibol e caçador (ou jogo de pega-pega com bola) foi significativamente mais freqüente nas meninas do que nos meninos. Outras atividades, como tênis de mesa e ciclismo foram relatadas por menos de $1 \%$ dos adolescentes.

A Tabela 2 apresenta variáveis independentes importantes para a compreensão dos níveis de atividade física. A amostra foi composta por $48,7 \%$ de meninos; $3,6 \%$ e $7,3 \%$ dos adolescentes foram classificados nos níveis sócio-econômicos A e E, respectivamente. Apenas 7,4\% dos adolescentes moravam em apartamento e 10,8\% estudavam em escolas particulares. Cerca de
$80 \%$ das mães não realizaram atividade física no lazer na semana anterior à entrevista. A prática de atividade física no lazer foi seis vezes mais comum entre as mães do nível A do que entre aquelas do nível $\mathrm{E}$ (dados não apresentados). A prevalência de obesidade (IMC $\geq 30 \mathrm{~kg} / \mathrm{m}^{2}$ ) materna foi de $22,7 \%$. O IMC médio nos adolescentes foi de $18,6 \mathrm{~kg} / \mathrm{m}^{2}$ (DP 3,6). A média diária de tempo gasto assistindo à televisão foi de 3,3 horas (DP 2,0). O percentual de adolescentes que relataram jogar vídeo-game por uma hora ou mais por dia foi de $22,4 \%$, enquanto $9,7 \%$ dos adolescentes relataram que usam o computador por uma hora ou mais por dia.

Entre todas as crianças, $99,1 \%$ participavam de atividades curriculares de educação física dentro da escola; $13,6 \%$ participam de alguma atividade física com instrutor (equipes, escolinhas, ginástica, dança) dentro da escola; $15,8 \%$ em outras atividades físicas com instrutor fora da escola. Quase $80 \%$ dos adolescentes praticaram alguma atividade física no lazer na semana anterior à entrevista e 72,8\% usavam regularmente um modo de deslocamento ativo para a escola.

A Tabela 2 mostra também a prevalência de diferentes tipos de atividade física conforme variáveis independentes; uma vez que praticamente todas as crianças realizavam aulas curriculares de educação física, esta variável não é mostrada. A participação em atividade física com instrutor na escola foi mais freqüente entre as meninas, e em adolescentes de nível sócio-econômico alto, entre aqueles que moravam 
Percentual de realização de diferentes tipos de atividades físicas no lazer na semana anterior à entrevista em meninos e meninas.

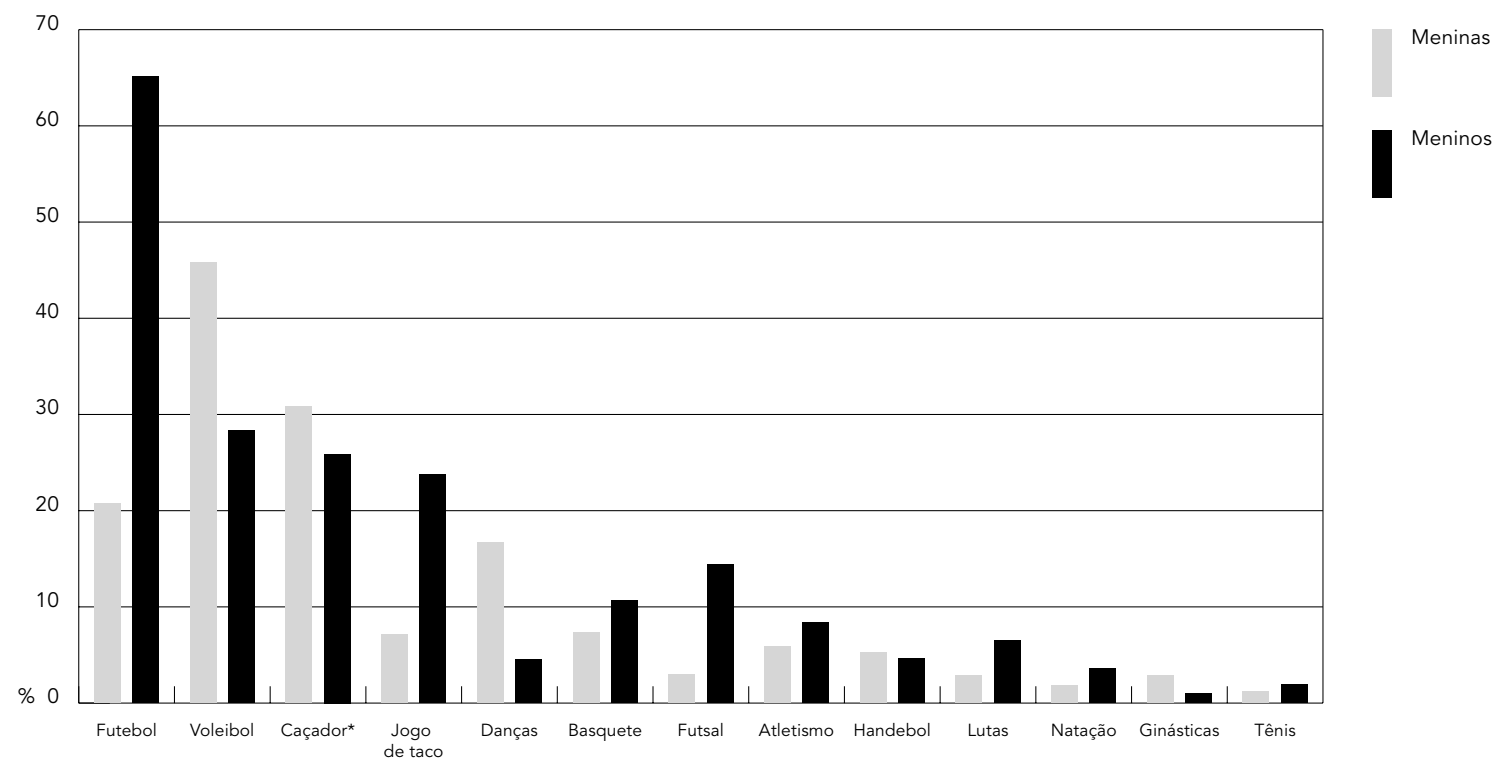

* Jogo de pega-pega com bola.

em apartamento, estudavam em escola particular, tinham mães ativas e com menor IMC, despendiam menos tempo assistindo à televisão, jogavam vídeo-game e usavam o computador por mais tempo.

Os fatores associados à participação em atividade física com instrutor fora da escola foram semelhantes aos relacionados com a prática de atividade física com instrutor na escola. As diferenças observadas foram uma maior freqüência entre meninos, ausência de associação com o tipo de escola e um efeito linear da prática de vídeo game.

A prática de atividade física no lazer (incluindo atividades com e sem instrutor) foi mais freqüente entre os meninos, de nível sócio-econômico alto, filhos de mães ativas e com menor IMC. Quanto maior o tempo de uso de computador e vídeo-game, maior o percentual de atividade física no lazer. Ao contrário, quanto menor o tempo assistindo à televisão, maior a prevalência de atividade física no lazer.

O deslocamento ativo para a escola foi mais comum nos jovens de nível sócio-econômico baixo, que moravam em casa, estudavam em escolas municipais ou estaduais, e que tinham menor IMC. Filhos de mães inativas e obesas apresentaram maior probabilidade de deslocamento ativo para o colégio. O tempo diário de uso de computador esteve associado inversamente com o deslocamento ativo.

Algumas das associações apresentadas na Tabela 2 não se mantiveram significativas após análise ajustada por regressão de Poisson (dados não apresentados em tabela). Para o desfecho atividade física com instrutor na escola, os efeitos do tipo de moradia e IMC da mãe deixaram de ser significativos. Em termos do desfecho atividade física com instrutor fora da escola, as variáveis atividade física da mãe no lazer, tempo diário de uso de computador e vídeogame perderam significância após ajuste. Para o deslocamento ativo, os efeitos do IMC e da inatividade física da mãe deixaram de ser significativos na análise multivariável.

A prática de atividade física com instrutor dentro ou fora da escola - esteve associada positivamente com a prática de atividade física sem instrutor, mas negativamente com o deslocamento ativo (ambos com $\mathrm{p}<0,001$ ). Não houve associação entre a prática de atividade física no lazer e o deslocamento ativo ( $\mathrm{p}=0,33$ ).

Como forma de agrupar os domínios da atividade física investigados, um escore foi cons- 
Distribuição da amostra conforme categorias das variáveis independentes e prática de atividade física em diferentes domínios.

\begin{tabular}{|c|c|c|c|c|c|}
\hline \multirow[t]{2}{*}{ Variável } & \multirow[t]{2}{*}{$\mathbf{N}$} & \multicolumn{2}{|c|}{$\begin{array}{l}\text { Atividade física com } \\
\text { instrutor \% (IC95\%) }\end{array}$} & \multirow{2}{*}{$\begin{array}{l}\text { Atividade física } \\
\text { total no lazer } \\
\text { \% (IC } 55 \%)\end{array}$} & \multirow{2}{*}{$\begin{array}{c}\text { Deslocamento } \\
\text { ativo } \\
\%(I C 95 \%)\end{array}$} \\
\hline & & Escola & Fora da escola & & \\
\hline Sexo & & $p=0,03$ & $p<0,001$ & $p<0,001$ & $p=0,20$ \\
\hline Masculino & 2.167 & $12,5(11,1-13,9)$ & $19,8(18,2-21,5)$ & $85,4(83,8-86,9)$ & $73,7(71,8-75,5)$ \\
\hline Feminino & 2.283 & $14,7(13,2-16,1)$ & $12,1(10,7-13,4)$ & $72,9(71,0-74,7)$ & $72,0(70,1-73,8)$ \\
\hline Nível sócio-econômico & & $p<0,001^{*}$ & $p<0,001^{*}$ & $p<0,001^{*}$ & $p<0,001 *$ \\
\hline A & 157 & $46,5(38,6-54,4)$ & $28,9(21,7-36,0)$ & $84,5(78,8-90,3)$ & $14,0(8,5-19,5)$ \\
\hline B & 645 & $19,2(16,2-22,3)$ & $19,3(16,2-23,3)$ & $81,6(78,5-84,6)$ & $48,1(44,3-52,0)$ \\
\hline $\mathrm{C}$ & 1.454 & $11,7(10,0-13,3)$ & $15,2(13,4-17,1)$ & $79,6(77,4-81,7)$ & $73,7(71,4-76,0)$ \\
\hline D & 1.761 & $10,0(8,6-11,4)$ & $13,9(12,3-15,6)$ & $77,6(75,6-79,5)$ & $85,1(83,4-86,8)$ \\
\hline$E$ & 315 & $13,0(9,3-16,8)$ & $14,9(11,0-18,9)$ & $76,7(71,8-81,3)$ & $86,3(82,5-90,2)$ \\
\hline Tipo de moradia & & $p<0,001$ & $p=0,001$ & $p=0,27$ & $p<0,001$ \\
\hline Casa & 4.095 & $13,0(12,0-14,0)$ & $15,3(14,2-16,4)$ & $79,2(77,9-80,4)$ & $74,6(73,2-75,9)$ \\
\hline Apartamento & 328 & $20,8(16,4-25,2)$ & $22,4(17,8-26,9)$ & $76,6(71,9-81,2)$ & $50,9(45,5-56,4)$ \\
\hline Tipo de escola & & $p<0,001$ & $p=0,18$ & $p=0,26$ & $p<0,001$ \\
\hline Municipal & 1.958 & $12,1(10,7-13,6)$ & $15,7(14,1-17,3)$ & $78,1(76,2-80,0)$ & $78,9(77,0-80,7)$ \\
\hline Estadual & 1.955 & $11,4(10,0-12,8)$ & $15,4(13,8-17,0)$ & $79,7(77,9-81,5)$ & $76,9(75,0-78,7)$ \\
\hline Particular & 474 & $29,4(25,3-33,5)$ & $18,8(15,3-22,4)$ & $81,1(77,5-84,7)$ & $34,0(30,0-38,2)$ \\
\hline Atividade física no lazer da mãe & & $p<0,001$ & $p=0,04$ & $p=0,01$ & $p<0,001$ \\
\hline Inativa & 3.536 & $12,4(11,3-13,4)$ & $15,3(14,1-16,4)$ & $78,2(76,8-79,6)$ & $75,2(73,8-76,7)$ \\
\hline Ativa & 887 & $18,4(15,8-20,9)$ & $18,1(15,5-20,6)$ & $82,1(79,5-84,7)$ & $63,2(60,0-66,3)$ \\
\hline $\begin{array}{l}\text { Índice de Massa Corporal } \\
\text { da mãe }\left(\mathrm{kg} / \mathrm{m}^{2}\right)\end{array}$ & & $p=0,01^{*}$ & $p=0,01^{*}$ & $p=0,02^{*}$ & $p=0,01^{*}$ \\
\hline$<25,0$ & 1.796 & $15,0(13,3-16,6)$ & $17,6(15,8-19,3)$ & $81,4(79,5-83,2)$ & $72,0(69,9-74,1)$ \\
\hline $25,0-29,9$ & 1.409 & $13,2(11,5-15,0)$ & $15,0(13,1-16,8)$ & $77,5(75,3-79,7)$ & $70,3(67,9-72,7)$ \\
\hline$\geq 30,0$ & 941 & $11,6(9,5-13,6)$ & $13,8(11,6-16,0)$ & $78,0(75,3-80,7)$ & $77,7(75,1-80,4)$ \\
\hline $\begin{array}{l}\text { Índice de Massa Corporal } \\
\text { do adolescente }\end{array}$ & & $p=0,34^{*}$ & $p=0,76^{*}$ & $p=0,16^{*}$ & $p<0,001^{*}$ \\
\hline 1으 tercil & 1.476 & $12,3(10,6-14,0)$ & $15,7(13,8-17,5)$ & $79,4(77,3-81,5)$ & $74,6(72,4-76,8)$ \\
\hline 2o tercil & 1.475 & $15,1(13,2-16,9)$ & $15,7(13,9-17,6)$ & $80,4(78,3-82,5)$ & $74,0(71,7-76,2)$ \\
\hline 3으 tercil & 1.475 & $13,5(11,8-15,2)$ & $16,1(14,2-18,0)$ & $77,3(75,1-79,4)$ & $69,9(67,5-72,2)$ \\
\hline Tempo diário assistindo à televisão & & $p=0,01^{*}$ & $p=0,01^{\star}$ & $p<0,001^{*}$ & $p=0,11^{\star}$ \\
\hline 1으 tercil & 1.579 & $15,0(13,2-16,7)$ & $17,5(15,7-19,4)$ & $81,9(80,0-83,8)$ & $72,3(70,0-74,5)$ \\
\hline 2o tercil & 1.401 & $14,1(12,2-15,9)$ & $15,9(14,0-17,9)$ & $78,9(76,7-81,1)$ & $71,2(68,8-73,6)$ \\
\hline 3o tercil & 1.457 & $11,8(10,1-13,5)$ & $13,9(12,1-15,6)$ & $75,9(73,6-78,1)$ & $74,9(72,7-77,1)$ \\
\hline Tempo diário jogando vídeo-game & & $p=0,02^{\star}$ & $p<0,001^{\star}$ & $p<0,001^{*}$ & $p=0,13^{\star}$ \\
\hline Não joga regularmente & 2.504 & $12,0(10,8-13,3)$ & $13,9(12,5-15,3)$ & $75,1(73,4-76,8)$ & $74,6(72,9-76,3)$ \\
\hline$<1$ hora por dia & 939 & $17,6(15,1-20,0)$ & $17,3(14,8-19,7)$ & $82,7(80,3-85,2)$ & $67,5(64,4-70,5)$ \\
\hline$\geq 1$ hora por dia & 992 & $14,0(11,8-16,2)$ & $19,2(16,7-21,6)$ & $85,5(83,3-87,7)$ & $73,6(70,8-76,3)$ \\
\hline Tempo diário no computador & & $p<0,001^{*}$ & $p<0,001 *$ & $p=0,01^{\star}$ & $p<0,001 *$ \\
\hline Não usa regularmente & 3.451 & $10,9(9,9-12,0)$ & $14,8(13,6-16,0)$ & $78,0(76,5-79,4)$ & $79,5(78,2-80,9)$ \\
\hline$<1$ hora por dia & 561 & $20,1(16,8-23,5)$ & $19,5(16,2-22,8)$ & $82,6(79,5-85,8)$ & $53,7(49,5-57,8)$ \\
\hline$\geq 1$ hora por dia & 430 & $26,7(22,5-30,9)$ & $19,1(15,3-22,8)$ & $82,3(78,6-86,0)$ & $44,2(39,5-48,9)$ \\
\hline
\end{tabular}

* Teste para tendência linear;

** Inclui atividade física com e sem instrutor. 
truído como o tempo semanal total despendido em atividade física, incluindo as categorias descritas acima. A prevalência de sedentarismo (<300 minutos por semana) foi de $58,2 \%$ (IC95\%: 56,7-59,7). A Tabela 3 mostra sua distribuição conforme as variáveis independentes, além das razões de prevalência brutas e ajustadas para as categorias das exposições estudadas. Na análise bruta, o sedentarismo esteve associado positivamente ao sexo feminino, nível sócio-econômico, estudar em escola particular, IMC do adolescente e tempo diário assistindo à televisão. A associação foi inversa para o tempo diário jogando vídeo-game. $\mathrm{Na}$ análise multivariável, o tipo de escola e o IMC do adolescente perderam a significância, enquanto a inatividade física da mãe no lazer mostrou-se um fator de risco para sedentarismo nos adolescentes.

\section{Discussão}

No presente estudo, com delineamento transversal aninhado a uma coorte de base populacional, o nível de atividade física de adolescentes de 10-12 anos de idade foi avaliado. Utilizando-se o ponto de corte recomendado para adolescentes 17 , a prevalência de sedentarismo foi elevada, indicando um amplo potencial de intervenção. A identificação de subgrupos populacionais com maior freqüência de sedentarismo pode guiar estratégias efetivas que visem a aumentar o nível de atividade física de adolescentes.

Algumas limitações deste estudo devem ser consideradas. Primeiro, apesar de os participantes fazerem parte de um estudo longitudinal prospectivo sobre saúde, a natureza dos dados aqui apresentados é transversal, visto que todas as informações foram coletadas em um único momento. Sendo assim, deve-se levar em conta a possibilidade de causalidade reversa na associação de atividade física com IMC e com o tempo despendido na televisão, vídeogame ou computador. O instrumento utilizado não foi submetido a um processo de validação em comparação a um método de referência. Como nenhum questionário de atividade física previamente validado em adolescentes brasileiros avalia diversos domínios da atividade física (deslocamento para a escola, atividade física com ou sem instrutor, dentro e fora da escola), foi necessário criar um novo instrumento. Os resultados do estudo piloto auxiliaram na redação das perguntas com o intuito de adequá-las à faixa etária em estudo. Apesar da falta de validação formal do questionário, a com- preensão das perguntas foi muito boa. Sugerese que estudos futuros comparem o questionário com métodos diretos de mensuração de atividade física, por exemplo, acelerômetros ou pedômetros.

Entre as vantagens do estudo, pode-se destacar a representatividade da população, incluindo adolescentes escolares e não-escolares, pertencentes aos diversos níveis sócio-econômicos da população. O percentual de perdas de acompanhamento foi baixo para um estudo longitudinal com mais de dez anos de duração, e não variou significativamente por sexo ou idade materna. O maior porcentual de perdas nos níveis sócio-econômicos mais altos justifica-se pela dificuldade na obtenção de listas com endereços dos alunos das escolas particulares.

A prevalência de sedentarismo entre os meninos (49\%) foi mais alta do que a observada em estudo com adolescentes de 15-19 anos da mesma cidade (22\%) 12, e mais baixa que a observada em pesquisa com adolescentes de 1415 anos de Niterói (85\%) 11. Entre as meninas, o percentual encontrado no presente estudo (67\%) também foi maior do que o observado no outro estudo realizado em Pelotas (55\%) $12 \mathrm{e}$ menor do que o observado em Niterói (94\%) 11. A análise dessas diferenças deve considerar que o ponto de corte para definição de sedentarismo foi diferente nesses estudos. No estudo de Niterói 11, sedentarismo foi definido como escore abaixo de três em uma escala que variava de zero a cinco pontos, incluindo atividades esportivas realizadas no tempo de lazer. No outro estudo realizado em Pelotas 12 , sedentarismo foi definido como menos de vinte minutos de atividade física, pelo menos três vezes por semana. No presente estudo, optou-se por utilizar a recomendação atual de atividade física para adolescentes 17, exatamente para facilitar comparações futuras. Caso as aulas de educação física fossem incluídas no escore total de atividade física, a prevalência de sedentarismo cairia de $58,2 \%$ para $48,4 \%$. Além das diferenças nos pontos de corte e instrumentos utilizados, questões ambientais, culturais e sazonalidade podem explicar parte das diferenças observadas nesses estudos.

A avaliação de várias esferas da atividade física na adolescência fornece dados inéditos sobre os diferentes padrões de atividade física nesses domínios. Por exemplo, o deslocamento ativo para a escola se associou com nível sócioeconômico baixo. Esse resultado é provavelmente explicado pela ausência de outra alternativa de transporte, ao invés de real consciência da população sobre os benefícios do deslocamento ativo. Por outro lado, o uso da bicicle- 
Prevalência e razões de prevalência (RP) de sedentarismo conforme variáveis independentes: análises bruta e ajustada.

\begin{tabular}{|c|c|c|c|}
\hline \multirow[t]{2}{*}{ Variável } & \multicolumn{2}{|c|}{ Análise bruta } & \multirow{2}{*}{$\begin{array}{l}\text { Análise ajustada } \\
\text { RP (IC95\%) }\end{array}$} \\
\hline & $\begin{array}{c}\text { Sedentarismo } \\
\% \text { (IC95\%) }\end{array}$ & RP (IC95\%) & \\
\hline Sexo & \multicolumn{2}{|c|}{$p<0,001$} & $p<0,001$ \\
\hline Masculino & $49,0(46,8-51,1)$ & 1,00 & 1,00 \\
\hline Feminino & $67,0(65,1-69,0)$ & $1,37(1,30-1,44)$ & $1,38(1,31-1,46)$ \\
\hline Nível sócio-econômico & \multicolumn{2}{|c|}{$p<0,001^{*}$} & $p<0,001^{\star}$ \\
\hline A & $63,9(56,2-71,5)$ & $1,26(1,07-1,49)$ & $1,27(1,08-1,49)$ \\
\hline B & $63,7(59,9-67,5)$ & $1,26(1,11-1,43)$ & $1,27(1,12-1,43)$ \\
\hline $\mathrm{C}$ & $57,9(55,3-60,5)$ & $1,15(1,02-1,29)$ & $1,14(1,01-1,28)$ \\
\hline $\mathrm{D}$ & $57,0(54,6-59,4)$ & $1,13(1,00-1,27)$ & $1,12(1,00-1,26)$ \\
\hline$E$ & $50,5(44,8-56,2)$ & 1,00 & 1,00 \\
\hline Tipo de moradia & \multicolumn{2}{|c|}{$p=0,38$} & $p=0,83$ \\
\hline Casa & $58,0(56,5-59,5)$ & 1,00 & 1,00 \\
\hline Apartamento & $60,5(55,1-65,9)$ & $1,04(0,95-1,14)$ & $0,99(0,90-1,09)$ \\
\hline Tipo de escola & \multicolumn{2}{|c|}{$p=0,003$} & $p=0,24$ \\
\hline Municipal & $56,3(54,1-58,6)$ & 1,00 & 1,00 \\
\hline Estadual & $57,9(55,7-60,1)$ & $1,03(0,97-1,09)$ & $1,01(0,95-1,07)$ \\
\hline Particular & $65,2(60,8-69,5)$ & $1,16(1,07-1,25)$ & $1,08(0,99-1,18)$ \\
\hline Atividade física no lazer da mãe & \multicolumn{2}{|c|}{$p=0,15$} & $p=0,04$ \\
\hline Inativa & $58,7(57,1-60,4)$ & $1,05(0,98-1,12)$ & $1,07(1,00-1,15)$ \\
\hline Ativa & $56,0(52,7-59,3)$ & 1,00 & 1,00 \\
\hline Índice de Massa Corporal da mãe $\left(\mathrm{kg} / \mathrm{m}^{2}\right)$ & \multicolumn{2}{|c|}{$p=0,98^{*}$} & $p=0,82^{*}$ \\
\hline$<25,0$ & $57,1(54,7-59,4)$ & 1,00 & 1,00 \\
\hline $25,0-29,9$ & $60,0(57,4-62,6)$ & $1,05(0,99-1,12)$ & $1,05(0,99-1,11)$ \\
\hline$\geq 30,0$ & $56,4(53,2-59,6)$ & $0,99(0,92-1,06)$ & $1,00(0,93-1,07)$ \\
\hline Índice de Massa Corporal do adolescente & \multicolumn{2}{|c|}{$p=0,04^{*}$} & $p=0,21^{*}$ \\
\hline 1으 tercil & $57,0(54,4-59,5)$ & 1,00 & 1,00 \\
\hline 2o tercil & $56,6(54,0-59,2)$ & $0,99(0,93-1,06)$ & $0,99(0,93-1,05)$ \\
\hline 3o tercil & $60,9(58,3-63,3)$ & $1,07(1,00-1,13)$ & $1,04(0,98-1,11)$ \\
\hline Tempo diário assistindo à televisão & \multicolumn{2}{|c|}{$p<0,001^{*}$} & $p=0,003^{*}$ \\
\hline 1으 tercil & $55,7(53,0-58,0)$ & 1,00 & 1,00 \\
\hline 2o tercil & $57,7(55,1-60,3)$ & $1,04(0,97-1,11)$ & $1,02(0,96-1,09)$ \\
\hline 3으 tercil & $61,6(59,1-64,2)$ & $1,11(1,04-1,18)$ & $1,10(1,03-1,17)$ \\
\hline Tempo diário jogando vídeo-game & \multicolumn{2}{|c|}{$p<0,001^{*}$} & $\mathrm{p}<0,001^{*}$ \\
\hline Não joga regularmente & $62,3(60,4-64,3)$ & 1,00 & 1,00 \\
\hline$<1$ hora por dia & $57,4(54,2-60,7)$ & $0,92(0,86-0,98)$ & $0,96(0,90-1,02)$ \\
\hline$\geq 1$ hora por dia & $48,2(45,0-51,4)$ & $0,77(0,72-0,83)$ & $0,83(0,77-0,89)$ \\
\hline Tempo diário no computador & \multicolumn{2}{|c|}{$p=0,06^{*}$} & $p=0,56^{*}$ \\
\hline Não usa regularmente & $57,4(55,7-59,1)$ & 1,00 & 1,00 \\
\hline$<1$ hora por dia & $60,4(53,3-64,5)$ & $1,05(0,98-1,13)$ & $1,00(0,93-1,09)$ \\
\hline$\geq 1$ hora por dia & $61,5(56,8-66,2)$ & $1,07(0,99-1,16)$ & $1,03(0,94-1,13)$ \\
\hline
\end{tabular}

* Teste para tendência linear. 
ta foi extremamente baixo, o que chama a atenção em uma cidade onde $27 \%$ dos trabalhadores adultos do sexo masculino utilizam bicicleta para ir ao trabalho 22. O resultado entre os adolescentes pode ser devido ao fato de que as escolas normalmente não oferecem segurança para que as bicicletas fiquem guardadas durante a aula, além da insegurança no trajeto tanto em relação à violência quanto ao desrespeito ao ciclista no trânsito. Além disso, muitos adolescentes podem utilizar outros modos de transporte para a escola por não terem uma bicicleta própria. O uso de ônibus foi maior nos níveis sócio-econômicos intermediários. Possivelmente, os mais ricos não usam o ônibus porque preferem o deslocamento de carro ou moto, enquanto os mais pobres não o utilizam devido ao preço da passagem ou a proximidade entre a residência e a escola.

Dados da Pesquisa de Padrões de Vida 23 sobre atividade física em adultos indicaram que os homens tendem a praticar com maior freqüência esportes coletivos (principalmente futebol), enquanto as mulheres preferem a caminhada e outras atividades não-esportivas. Em adolescentes de 10-12 anos de idade, os meninos confirmaram seu interesse pelo futebol, enquanto as meninas praticaram o voleibol com maior freqüência. Duas atividades recreativas não formais - o jogo de caçador (ou jogo de pega-pega com bola) e o jogo de taco - foram relatadas com freqüência, indicando que nessa faixa etária a busca por atividades físicas lúdicas ainda é muito comum, além do fato de que nessa idade atividades físicas que congreguem meninos e meninas ainda são bem aceitas. Estudo brasileiro prévio 11 mostrou que $75 \%$ dos adolescentes masculinos praticavam o futebol, valor comparável aos $65 \%$ encontrados no presente estudo. Entre as meninas, a dança foi relatada por $60 \%$ delas no estudo realizado em Niterói e por apenas $17 \%$ no presente estudo. Essa diferença pode ser explicada pela faixa etária dos dois estudos, visto que o estudo de Niterói incluiu adolescentes de 14-15 anos de idade.

Os diferentes padrões na prática de atividade física com instrutor e deslocamento ativo conforme nível sócio-econômico merecem destaque. Enquanto os mais ricos tendem a praticar com maior freqüência atividade física com instrutor, a freqüência de deslocamento ativo nesse grupo é muito baixa. Apenas $14 \%$ dos adolescentes do nível A usam a caminhada no deslocamento para a escola e nenhum relatou o uso da bicicleta nesse deslocamento. Preocupações com segurança podem explicar em parte esse achado.
A prevalência global de sedentarismo, agrupando-se todos os domínios, foi maior entre os níveis sócio-econômicos altos, resultado contrário ao encontrado para atividade física de lazer em estudo realizado também em Pelotas 12. Os dados do presente estudo podem explicar essa diferença, visto que o maior nível de atividade física no deslocamento para a escola entre os pobres não foi considerado no estudo anterior.

Estudos anteriores já identificaram que o nível de atividade física da mãe é um fator associado ao nível de atividade física das crianças 24 . No presente estudo, houve uma associação entre nível de atividade física da mãe no lazer e sedentarismo dos filhos na adolescência. O tempo diário assistindo à televisão na adolescência tem se mostrado associado com desfechos desfavoráveis na própria adolescência 25 e até mesmo na idade adulta 26 . Neste estudo, houve relação positiva entre número de horas diárias assistindo à televisão e sedentarismo. No entanto, um maior número de horas jogando vídeo-game se mostrou positivamente associado ao nível de atividade física. Uma das hipóteses deste estudo era que adolescentes que moram em apartamento teriam um menor nível de atividade física do que aqueles que moram em casa. No entanto, após controle para nível sócio-econômico e tipo de escola, não foram observadas diferenças no nível de atividade física entre jovens que moram em apartamento ou em casa.

A maioria dos estudos agrupa o tempo diário despendido na televisão, computador ou vídeo-game em uma única variável, o que impossibilita avaliar o efeito separado de cada um desses indicadores. Uma possível explicação para o efeito do vídeo-game é que grande parte dos jogos é de lutas e esportes, o que poderia servir como estímulo à prática de atividade física entre os jovens. Na população estudada, a participação em esportes de lutas foi de 3,9\% nos jovens que não usam vídeo-game regularmente, 5,3\% naqueles que usam o vídeo-game por menos de uma hora por dia e $6,2 \%$ naqueles que usam o vídeo-game por uma hora ou mais por dia. Uma hipótese é que o vídeo-game pode ser usado independentemente das condições climáticas (meses de frio, umidade e chuva), além de ser jogado tanto individualmente quanto em pequenos grupos, ao contrário do futebol, por exemplo. Outros estudos são necessários para explorar mais detalhadamente a associação entre sedentarismo e tempo diário jogando vídeo-game. O maior nível de atividade física dos meninos já foi amplamente mostrado e discutido em publicações anteriores 11,12,27,28. 
Mesmo ao somar todos os tipos de atividade física praticados dentro das escolas (aulas de educação física e a participação em escolinhas ou equipes esportivas, de ginástica ou de dança), a maioria dos adolescentes estudados não atinge as recomendações atuais quanto à prática de atividade física. Um estudo norteamericano 29 recente mostrou que o aumento do número semanal de aulas de educação física pode auxiliar no combate ao sobrepeso. $\mathrm{Da}$ mesma forma, tal aumento, somado a um au- mento da intensidade das aulas, poderia minimizar o grave quadro observado no presente estudo quanto à elevada prevalência de sedentarismo na adolescência.

Em resumo, a prevalência de sedentarismo encontrada nessa população é elevada. Estratégias efetivas de combate ao sedentarismo na adolescência são necessárias, pois além de afetar mais da metade dos jovens, o sedentarismo nessa faixa etária é um fator de risco para inatividade física na idade adulta 3,4 .

\section{Resumo}

A atividade física na adolescência acarreta vários benefícios à saúde, seja por uma influência direta sobre a morbidade na própria adolescência, seja por uma influência mediada pelo nível de atividade física na idade adulta. Avaliou-se a prevalência de sedentarismo e fatores associados em 4.452 adolescentes de 1012 anos de idade, participantes do Estudo de Coorte de Nascimentos de 1993 em Pelotas, Rio Grande do Sul, Brasil. Sedentarismo foi definido como $<300$ minutos por semana de atividade física. As perdas de acompanhamento desde o nascimento totalizaram 12,5\%. A prevalência de sedentarismo foi de 58,2\% (IC95\%: 56,7-59,7). Na análise multivariável, o sedentarismo se associou positivamente ao sexo feminino, ao nível sócio-econômico, a ter mãe inativa e ao tempo diário assistindo à televisão. O sedentarismo se associou negativamente com o tempo diário de uso de vídeo-game. Adolescentes de nível econômico baixo apresentaram maior freqüência de deslocamento ativo para a escola. Estratégias efetivas de combate ao sedentarismo na adolescência são necessárias devido à sua alta prevalência e sua associação com inatividade física na idade adulta.

Atividade Motora; Aptidão Física; Adolescente; Exercício

\section{Colaboradores}

P. C. Hallal coordenou o trabalho de campo, a análise e a escrita do artigo. A. D. Bertoldi participou nos processos de análise e redação do manuscrito. H. Gonçalves participou da coordenação do trabalho de campo e da escrita do artigo. C. G. Victora revisou o trabalho desde a sua concepção até as fases de análise e redação.

\section{Agradecimentos}

Esta análise foi financiada pela iniciativa denominada Major Awards for Latin America on Health Consequences of Population Change. Fases anteriores do Estudo de Coorte de Nascimentos de 1993 em Pelotas foram financiadas pela União Européia, pelo Programa de Apoio a Núcleos de Excelência, pelo Conselho Nacional de Desenvolvimento Científico e Tecnológico e pelo Ministério da Saúde. 


\section{Referências}

1. Twisk JW. Physical activity guidelines for children and adolescents: a critical review. Sports Med 2001; 31:617-27.

2. Gordon-Larsen P, Nelson MC, Popkin BM. Longitudinal physical activity and sedentary behavior trends: adolescence to adulthood. Am J Prev Med 2004; 27:277-83.

3. Kraut A, Melamed S, Gofer D, Froom P. Effect of school age sports on leisure time physical activity in adults: The CORDIS Study. Med Sci Sports Exerc 2003; 35:2038-42.

4. Tammelin T, Nayha S, Hills AP, Jarvelin MR. Adolescent participation in sports and adult physical activity. Am J Prev Med 2003; 24:22-8.

5. Parsons TJ, Power C, Logan S, Summerbell CD. Childhood predictors of adult obesity: a systematic review. Int J Obes Relat Metab Disord 1999; 23 Suppl 8:S1-107.

6. Centers for Disease Control and Prevention. Physical activity levels among children aged 9-13 years: United States, 2002. Atlanta: Centers for Disease Control and Prevention; 2002.

7. Klasson-Heggebo L, Anderssen SA. Gender and age differences in relation to the recommendations of physical activity among Norwegian children and youth. Scand J Med Sci Sports 2003; 13:293-8.

8. LeonGuerrero RT, Workman RL. Physical activity and nutritional status of adolescents on Guam. Pac Health Dialog 2002; 9:177-85.

9. Monge-Rojas R, Nunez HP, Garita C, Chen-Mok M. Psychosocial aspects of Costa Rican adolescents' eating and physical activity patterns. J Adolesc Health 2002; 31:212-9.

10. Westerstahl M, Barnekow-Bergkvist M, Hedberg $\mathrm{G}$, Jansson E. Secular trends in body dimensions and physical fitness among adolescents in Sweden from 1974 to 1995 . Scand J Med Sci Sports 2003; 13:128-37.

11. Silva RCR, Malina RM. Nível de atividade física em adolescentes do Município de Niterói, Rio de Janeiro, Brasil. Cad Saúde Pública 2000; 16:1091-7.

12. Oehlschlaeger MH, Pinheiro RT, Horta B, Gelatti C, Santana P. Prevalência e fatores associados ao sedentarismo em adolescentes de área urbana. Rev Saúde Pública 2004; 38:157-63.

13. Guedes DP, Guedes JERP. Níveis de prática de atividade física habitual em adolescentes. Rev Bras Med Esporte 2001; 7:187-99.

14. Gomes VB, Siqueira KS, Sichieri R. Atividade física em uma amostra probabilística da população do Município do Rio de Janeiro. Cad Saúde Pública $2001 ; 17: 969-76$.

15. Victora CG, Barros FC, Halpern R, Menezes AM, Horta BL, Tomasi E, et al. Tendências e diferencias na saúde materno-infantil: delineamento e metodologia das coortes de 1982 e 1993 de mães e crianças de Pelotas, Rio Grande do Sul. Rev Saúde Pública 1996; 30:34-45.

16. Associação Nacional de Empresas de Pesquisa. Critério de classificação econômica Brasil. São Paulo: Associação Nacional de Empresas de Pesquisa; 1996.
17. Biddle S, Cavill N, Sallis J. Young and active? Young people and health-enhancing physical activity - evidence and implications. London: Health Education Authority; 1998.

18. Nader PR; National Institute of Child Health and Human Development Study of Early Child Care and Youth Development Network. Frequency and intensity of activity of third-grade children in physical education. Arch Pediatr Adolesc Med 2003; 157:185-90.

19. Cameron N. The measurement of human growth. London: Croom Helm; 1984.

20. Barros AJ, Hirakata VN. Alternatives for logistic regression in cross-sectional studies: an empirical comparison of models that directly estimate the prevalence ratio. BMC Med Res Methodol $2003 ; 3: 21$.

21. Victora CG, Huttly SR, Fuchs SC, Olinto MT. The role of conceptual frameworks in epidemiological analysis: a hierarchical approach. Int J Epidemiol 1997; 26:224-7.

22. Bacchieri G, Gigante DP, Assunção MC. Determinantes e padrões de utilização da bicicleta e acidentes de trânsito sofridos por ciclistas trabalhadores da Cidade de Pelotas. Cad Saúde Pública 2005; 21:1499-508.

23. Monteiro CA, Conde WL, Matsudo SM, Matsudo VR, Bonsenor IM, Lotufo PA. A descriptive epidemiology of leisure-time physical activity in Brazil, 1996-1997. Rev Panam Salud Pública 2003; 14:246-54.

24. Davison KK, Cutting TM, Birch LL. Parents' activity-related parenting practices predict girls' physical activity. Med Sci Sports Exerc 2003; 35:1589-95.

25. Proctor MH, Moore LL, Gao D, Cupples LA, Bradlee ML, Hood MY, et al. Television viewing and change in body fat from preschool to early adolescence: The Framingham Children's Study. Int J Obes Relat Metab Disord 2003; 27:827-33.

26. Hancox RJ, Milne BJ, Poulton R. Association between child and adolescent television viewing and adult health: a longitudinal birth cohort study. Lancet 2004; 364:257-62.

27. Aarnio M, Winter T, Peltonen J, Kujala UM, Kaprio J. Stability of leisure-time physical activity during adolescence - a longitudinal study among 16-, 17- and 18-year-old Finnish youth. Scand J Med Sci Sports 2002; 12:179-85.

28. Riddoch CJ, Bo-Andersen L, Wedderkopp N, Harro M, Klasson-Heggebo L, Sardinha LB, et al. Physical activity levels and patterns of 9- and 15-yr-old European children. Med Sci Sports Exerc 2004; 36:86-92.

29. Datar A, Sturm R. Physical education in elementary school and body mass index: evidence from the early childhood longitudinal study. Am J Public Health 2004; 94:1501-6.

Recebido em 08/Jul/2005

Versão final reapresentada em 29/Nov/2005

Aprovado em 12/Dez/2005 